\title{
Commentary: Can the esophagus curse a lung transplant?
}

\author{
Joseph B. Shrager, MD
}

Masuda and colleagues ${ }^{1}$ describe a beautifully executed study from a major lung transplantation center in which the authors use their own, granular data to address a substantial and unresolved clinical issue. The study is an outstanding example of bringing core physiological principles to bear in trying to understand an interesting and relevant problem. Routinely carrying out and then analyzing the results of high-resolution esophageal motility studies pre- and postoperatively in all lung transplant patients reflects an impressive, sustained commitment by this group to this area of investigation.

The authors extend their previous work in the area of esophageal dysfunction in patients with severe lung disease and following lung transplantation, and how these factors may affect lung transplantation outcomes. In previous publications, this group established that greater thoracoabdominal pressure gradient is associated with greater reflux in patients with severe lung disease, and that most patients achieve increased esophageal peristaltic vigor posttransplant. In their latest research, they set out to determine whether patients who have severely aperistaltic esophagus on pretransplant testing have a worse clinical course posttransplantwith the hypothesis that these patients might experience more reflux and thus potentially more bronchiolitis obliterans. In a subanalysis, they try to determine whether improved peristalsis that often occurs posttransplant is associated with reduction in the risk of a poor outcome.

The main findings are that approximately $10 \%$ of patients undergoing lung transplant present preoperatively with severe esophageal dysfunction, and that about two-thirds of these individuals see an improvement in this aperistalsis posttransplant. Further, they demonstrate that whereas those whose aperistalsis does not improve posttransplant have significantly decreased survival and likely increased rates of bronchiolitis obliterans syndrome, those whose aperistalsis does improve have equal survival to those who did not experience a severe

From the Division of Thoracic Surgery, Department of Cardiothoracic Surgery, Stanford University School of Medicine, Stanford, Calif.

Disclosure: Author has nothing to disclose with regard to commercial support.

Received for publication Feb 26, 2020; revisions received Feb 26, 2020; accepted for publication Feb 26, 2020; available ahead of print March 19, 2020.

Address for reprints: Joseph B. Shrager, MD, Stanford Hospital, Falk Building, 300

Pasteur Dr, Stanford, CA 94305-5407 (E-mail: shrager@stanford.edu).

J Thorac Cardiovasc Surg 2020;160:1628

$0022-5223 / \$ 36.00$

Copyright (C) 2020 by The American Association for Thoracic Surgery

https://doi.org/10.1016/j.jtcvs.2020.02.124

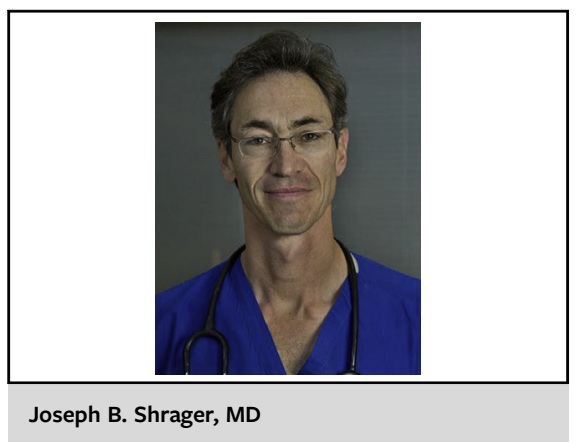

CENTRAL MESSAGE

Although most patients have

posttransplant recovery of

esophageal dysfunction, aperis-

taltic esophagus that persists

following lung transplant likely

bodes poorly for the lungs.

motility problem preoperatively. Many of those who did not have recovery of aperistalsis after transplant were patients with scerloderma.

The hypotheses for the work are compelling, the rationale strong, and the results convincing. The propensity matching on factors apart from esophageal dysfunction to compare outcomes between matched groups appears sound. The authors are careful not to overstep their data with overreaching conclusions.

The potential clinical significance of the work is obvious, particularly given our environment in which there is a shortage of donor lungs and the resulting impetus to avoid transplant in recipients who have a high risk of a poor outcome. First, although the authors intentionally, I think, avoid concluding that patients with scerloderma and aperistaltic esophagus should not undergo transplant, this study does suggest the need for future work to determine whether this is indeed the case. More broadly, one would like to see a more focused study to identify other predictors (beyond a diagnosis of scleroderma) that predict failure to recover peristaltic function postoperatively and therefore a poor outcome. Additionally, the study has implications for what might be the ideal approaches to peritransplant nutrition and antireflux interventions.

The authors are to be congratulated for this compelling piece of clinical research.

\section{Reference}

1. Masuda T, Mittal SK, Csucska M, Kovacs B, Walia R, Huang JL, et al. Esophageal aperistalsis and lung transplant: recovery of peristalsis after transplant is associated with improved long-term outcomes. J Thorac Cardiovasc Surg. 2020;160:1613-26. 